\title{
Toxicidade aguda e risco ecotoxicológico do inseticida tiametoxam para alevinos de tilápia-do-nilo
}

\author{
[Acute toxicity and ecotoxicological risk of thiamethoxam insecticide \\ in nile tilapia fingerlings] \\ A.C.L. Albinati ${ }^{1}$, R.C.B. Albinati ${ }^{2}$, A.D. Lira $^{3}$, P.C. Soares ${ }^{4}$ \\ ${ }^{1}$ Univasf - Petrolina, PE e Aluna de pós-graduação - Universidade Federal Rural \\ de Pernambuco -UFRPE - Recife, PE \\ ${ }^{2}$ EMEV - Universidade Federal da Bahia - UFBA - Salvador, BA \\ ${ }^{3}$ Aluna de pós-graduação -UFRGS - Porto Alegre, RS \\ ${ }^{4}$ Universidade Federal Rural de Pernambuco - UFRPE - Recife, PE
}

\begin{abstract}
RESUMO
O tiametoxam é um inseticida neonicotinóide usado em diversas culturas e classificado como perigoso para o meio ambiente.O objetivo do presente trabalho foi avaliar a toxicidade aguda do inseticida, por meio da determinação da CL50\%, e o risco ecotoxicológico com mensuração da concentração ambiental estimada (CAE) e do quociente de risco $(\mathrm{QR})$. O experimento foi realizado com alevinos de tilápias expostas a $150,300,450,600$ e $750 \mathrm{mg} / \mathrm{L}$ de $A$ ctara ${ }^{\circledR}$ WG por um período total de 96 horas. O oxigênio dissolvido, o $\mathrm{pH}$ e a temperatura foram mensurados diariamente em todos os aquários. Nos grupos experimentais, houve uma variação dos valores de $\mathrm{pH}$ e de OD para as diferentes concentrações do inseticida. A CL50\% 96h do Actara ${ }^{\circledR}$ para alevinos de tilápia foi de 322,08ppm. O quociente de risco (QR) variou de baixo a alto, de acordo com a metodologia usada.
\end{abstract}

Palavras-chave: ecotoxicologia, peixe, Actara, CL50\%

\begin{abstract}
The neonicotinoid insecticide thiamethoxam is used in different cultures and classified as dangerous for the environment. The aim of this study was to evaluate the acute toxicity of thiamethoxam by determining the lethal concentration (LC50) and ecotoxicological risk through Estimated Environmental Concentration (EEC) and Risk Quotient (RQ) measurement. The assays were done with Tilapia fingerlings exposed to 150, 300, 450, 600 and 750mg / L Actara WG during 96 hours. Dissolved oxygen, $\mathrm{pH}$ and temperature were measured daily in all aquariums. Dissolved oxygen and $\mathrm{pH}$ varied in the experimental groups. The LC50 Actara ${ }^{\circledR} ; 96 \mathrm{~h}$ was $322.08 \mathrm{ppm}$. The risk quotient (RQ) ranged from low to high according to the methodology used.
\end{abstract}

Keywords: ecotoxicology, fish, Actara, LC50

\section{INTRODUÇÃO}

No modelo agrícola atual, os agrotóxicos são tidos como indispensáveis, sendo também um dos principais poluentes químicos que se difundem pelo planeta (Grisolia, 2005). $\mathrm{Na}$ última década, o mercado de agrotóxicos no Brasil se expandiu rapidamente, num ritmo muito maior do que o apresentado pelo mercado

Recebido em 13 de agosto de 2015

Aceito em 18 de fevereiro de 2016

E-mail: catarina.albinati@gmail.com mundial, levando o Brasil a alcançar a posição de maior consumidor mundial de agrotóxicos desde 2008 (Londres, 2011; Rigotto, 2014). A frequência de uso também é maior que a média verificada em países de altas latitudes, em função do clima e dos métodos agrícolas utilizados (Grazziero, 2015). Apesar de úteis na agricultura, o seu amplo emprego tem perturbado o meio ambiente e organismos importantes ao ecossistema (U־gurlu, 2015). 
A base dos estudos de Toxicologia Aquática é formada pelo desenvolvimento principalmente de dois testes experimentais, os de toxicidade aguda e os de toxicidade crônica. Os testes de toxicidade aguda proporcionam rápidas respostas na estimativa dos efeitos letais de um agente tóxico sobre organismos aquáticos. Compreendem análises experimentais de curta duração (24-96 horas), que visam determinar a concentração letal média (CL50\%). Os testes de toxicidade aguda geram dados que servem de base para a elaboração de outros estudos subsequentes, como os testes de toxicidade crônica e as avaliações de risco ecotoxicológico (Lombardi, 2004).

O tiametoxam é um inseticida neonicotinóide de segunda geração que apresenta classificação toxicológica III (medianamente tóxico) e classe ambiental III (perigoso para o meio ambiente). O mecanismo de ação do tiametoxam é por ligação nos receptores nicotínicos da acetilcolina (Maiennfisch, 2001). Essa nova geração de inseticidas veio substituir os organofosforados e os carbamatos, os quais têm diminuído a sua eficiência devido à resistência dos insetos, ou ainda, vêm sendo menos utilizados, considerando-se o aumento das restrições de seu uso, bem como os aspectos toxicológicos no campo (Tavares, 2011). Pouco se sabe sobre sua toxicidade para os organismos de água doce não alvo e seu potencial efeito nos ecossistemas de água doce (Ǔgurlu, 2015).

Os produtos à base de tiametoxam são utilizados em diversas culturas, como: abacaxi, abobrinha, alface, arroz, batata, berinjela, café, cana-deaçúcar, citros, feijão-vagem, fumo, maçã, mamão, melancia, melão, morango, pepino, pêssego, pimentão, repolho, tomate e uva (Sistema..., 2012). Além do uso agrícola, o tiametoxam tem sido prospectado para uso em aquicultura. De acordo com Carraschi et al. (2014), o produto apresenta alta eficácia no controle de Anacanthorus penilabiatus em Pacus. Segundo levantamento realizado por Scorza Jr. e Rigatano (2012), a existência de alguns estudos sobre a degradação, sorção e lixiviação do tiametoxam, nas condições edafoclimáticas brasileiras, demonstra a alta persistência e a intensa lixiviação nos solos. Em estudo realizado por Silva et al. (2014), foi demonstrado que o tiametoxam é moderadamente persistente no solo e tem alto potencial de transporte quando dissolvido em água.

Alguns autores consideram o tiametoxam praticamente não tóxico para peixes (Maeinfisch, 2001), no entanto ainda são poucos os estudos realizados sobre os efeitos do tiametoxam com esses animais. Carraschi (2014), ao estudar os riscos ambientais e a ecotoxicologia do tiametoxam sobre Pacus (Piaractus mesopotamicus), relata que o produto é ligeiramente tóxico para a espécie estudada, que existe risco de intoxicação ambiental e que a presença do tiametoxam diminui o oxigênio dissolvido na água. Bose et al. (2011) observaram que em tilapias-do-nilo expostas a doses subletais de tiametoxam ocorreu um impacto significativo no crescimento dos animais, e distúrbios hematológicos foram observados por Roy e Nath (2011). Nesse contexto, torna-se importante o estudo dos efeitos do tiametoxam em peixes e, para isso, optou-se pela tilápia-do-nilo (Oreochromis niloticus), espécie exótica mais cultivada no Brasil. Dessa forma, o objetivo deste trabalho foi avaliar a toxicidade aguda do tiametoxam, por meio da determinação da concentração letal 50\% (CL50) para tilápias-do-nilo, e o risco ecotoxicológico de seu uso.

\section{MATERIAL E MÉTODOS}

O experimento conduzido foi aprovado pelo Comitê de Ética no Uso de Animais da Escola de Medicina Veterinária e Zootecnia da Universidade Federal da Bahia, sob o número 36/2014.

O experimento foi realizado com 240 alevinos de tilápias (Oreochromis niloticus), adquiridos de uma piscicultura comercial, com peso médio de $0,55 \pm 0,13 \mathrm{~g}$ e clinicamente hígidos. Os animais, ao chegarem, foram colocados em tanques de fibra de vidro de 500L, com aeração artificial, onde foram mantidos para aclimatação por 10 dias. Os animais foram alimentados com ração comercial própria para a espécie, três vezes ao dia, durante a aclimatação, sendo a alimentação suspensa 24 horas antes do início do experimento, de acordo com as recomendações das normas de ensaio de toxicidade aguda com peixes da Associação Brasileira de Normas Técnicas - ABNT (ABNT, 2011). 
$\mathrm{O}$ inseticida utilizado foi $\mathrm{o}$ Actara $250 \mathrm{WG}^{\circledR}$, produto comercial à base de tiametoxam, comercializado pela Syngenta Proteção de Cultivos Ltda. Na sua composição estão presentes $250 \mathrm{~g} / \mathrm{kg}$ de 3-(2-cloro-tiazol-5ilmetil)-5-metil-[1,3,5] oxadiazinan-4-ilideno-Nnitroamina e $750 \mathrm{~g} / \mathrm{kg}$ de ingredientes inertes.

Os parâmetros de qualidade da água, como oxigênio dissolvido, $\mathrm{pH}$ e temperatura, foram mensurados diariamente em todos os aquários. $\mathrm{O}$ oxigênio dissolvido foi medido com medidor de oxigênio dissolvido (Instrutherm MO-880); o $\mathrm{pH}$, por meio de medidor de $\mathrm{pH}$ digital portátil (Instrutherm pH-1700); a temperatura, com termômetro digital (TE-300).

O experimento foi realizado num período total de 96 horas, com observação e quantificação de mortalidade a cada 24 horas. Para tanto, foram utilizadas 24 caixas plásticas com 20 litros de água declorada, em sistema estático com aeração artificial. Foram utilizadas cinco diferentes concentrações do inseticida e um controle (sem inseticida), com quatro repetições por tratamento. As concentrações utilizadas foram 150, 300, 450, 600 e $750 \mathrm{mg} / \mathrm{L}$ de Actara WG. Foram utilizados 10 peixes por caixa, perfazendo um total de 40 peixes por tratamento. Com base na mortalidade diária, foi calculada a CL50\%. Os riscos ecotoxicológicos foram avaliados por meio do cálculo da concentração ambiental estimada (CAE) e do quociente de risco (QR). Para o cálculo da CAE, foram considerados os cenários de um reservatório de 1 ha de área, com $0,3 \mathrm{~m}$ e $2,0 \mathrm{~m}$ de profundidade, embasado em trabalho descrito por Manrique et al. (2013). Os cálculos foram realizados para uma diluição de $100 \%$ da maior dose recomendada na bula do Actara, que é de $2000 \mathrm{~g}$ i.a./ha. A CAE refere-se à razão entre $100 \%$ da maior dose recomendada $\mathrm{e}$ o volume total do reservatório. Para o cálculo do quociente de risco (QR), que é revelado pela razão entre CAE e CL50\%, foram utilizadas as CAEs calculadas conforme mencionadas anteriormente, assim como uma CAE de
$75 \mathrm{mg} / \mathrm{L}$, que representa a dose mais eficaz no controle de parasitas em Pacus (Carraschi et al., 2014). As CL50\% utilizadas foram baseadas no produto formulado, assim como na porcentagem de ingrediente ativo presente no produto $(25 \%)$. A classificação de risco foi baseada nos índices da Proposed... (1996).

Para a determinação da CL50\%, os dados de mortalidade observados foram avaliados com o programa estatístico Trimed Sperman Karber (Hamilton et al., 1977). Os dados foram analisados por meio do programa computacional Statistical Analysis System (SAS 2002), utilizando-se o procedimento GLM. Para todas as análises estatísticas realizadas foi adotado o nível de significância $(\mathrm{P})$ de $5 \%$. Nos casos em que houve significância no teste $\mathrm{F}$, as médias de $\mathrm{pH}$ e de oxigênio dissolvido na água (OD) foram submetidas a análises de regressão, por se tratar de dados contínuos. Realizou-se, também, análise de regressão para a mortalidade de alevinos em função da concentração do inseticida.

\section{RESULTADOS E DISCUSSÃO}

A qualidade da água manteve-se dentro da normalidade durante o experimento nos grupos controle, com um $\mathrm{pH}$ médio em torno de $8,1 \pm$ 0,1 ; oxigênio dissolvido (OD) de 7,1 $\pm 0,4 \mathrm{mg} / \mathrm{L}$ e uma temperatura de $27,4 \pm 0,3^{\circ} \mathrm{C}$. A temperatura, o OD e o $\mathrm{pH}$ da água, numa situação de conforto para peixes tropicais, deve estar entre $28-32^{\circ} \mathrm{C}$, superior a $5 \mathrm{mg} / \mathrm{L}$ e entre 6,5-8,0, respectivamente (Kubtiza e Kubtiza, 2000; Brasil, 2005). Nos grupos experimentais, houve uma variação dos valores de $\mathrm{pH}$ (Fig.1) e de OD (Fig.2) para as diferentes concentrações do inseticida, com valores de $\mathrm{pH}$ variando de 5,2 a 8,2 e de OD de 3,7 a 7,5mg/L, embora sem correlação linear significativa entre essas variáveis e sem que atingissem valores letais de $\mathrm{pH}$ ou de OD para os peixes. 


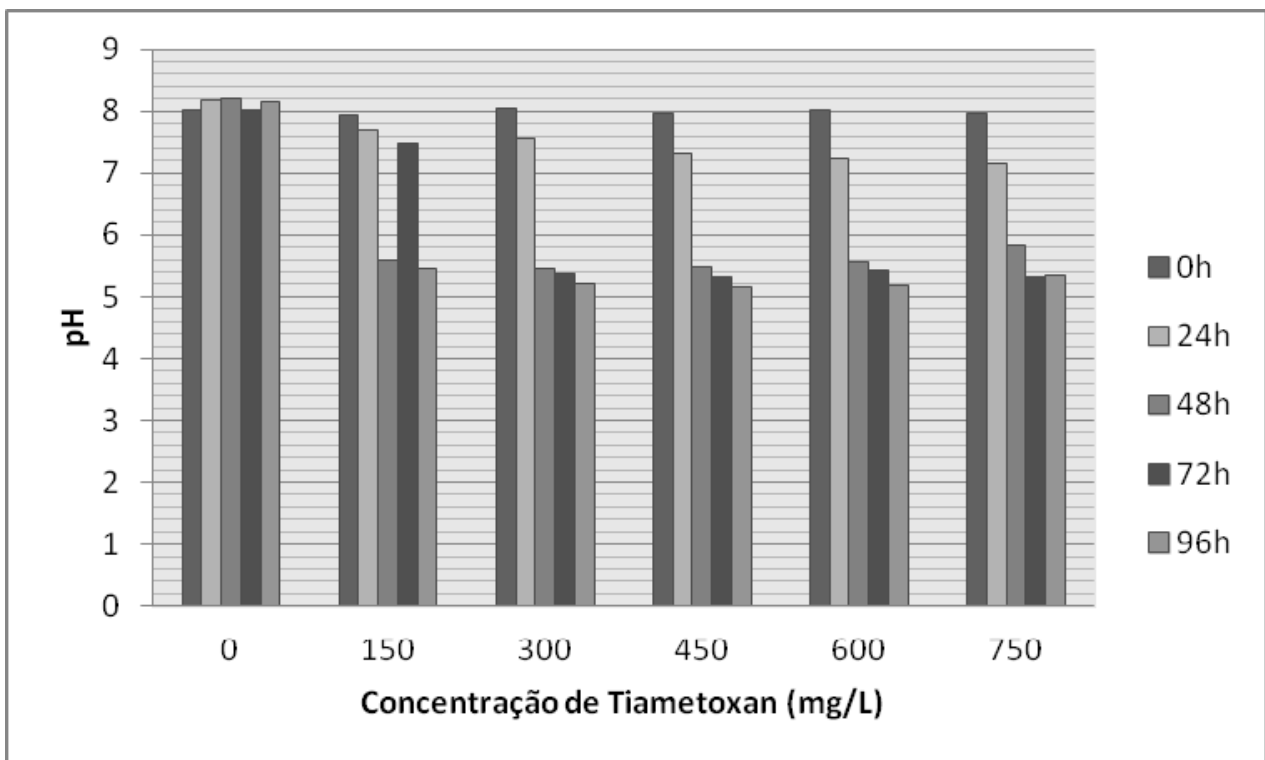

Figura 1. Variação do $\mathrm{pH}$ da água em função do tempo de exposição ao inseticida, para diferentes concentrações de tiametoxam.

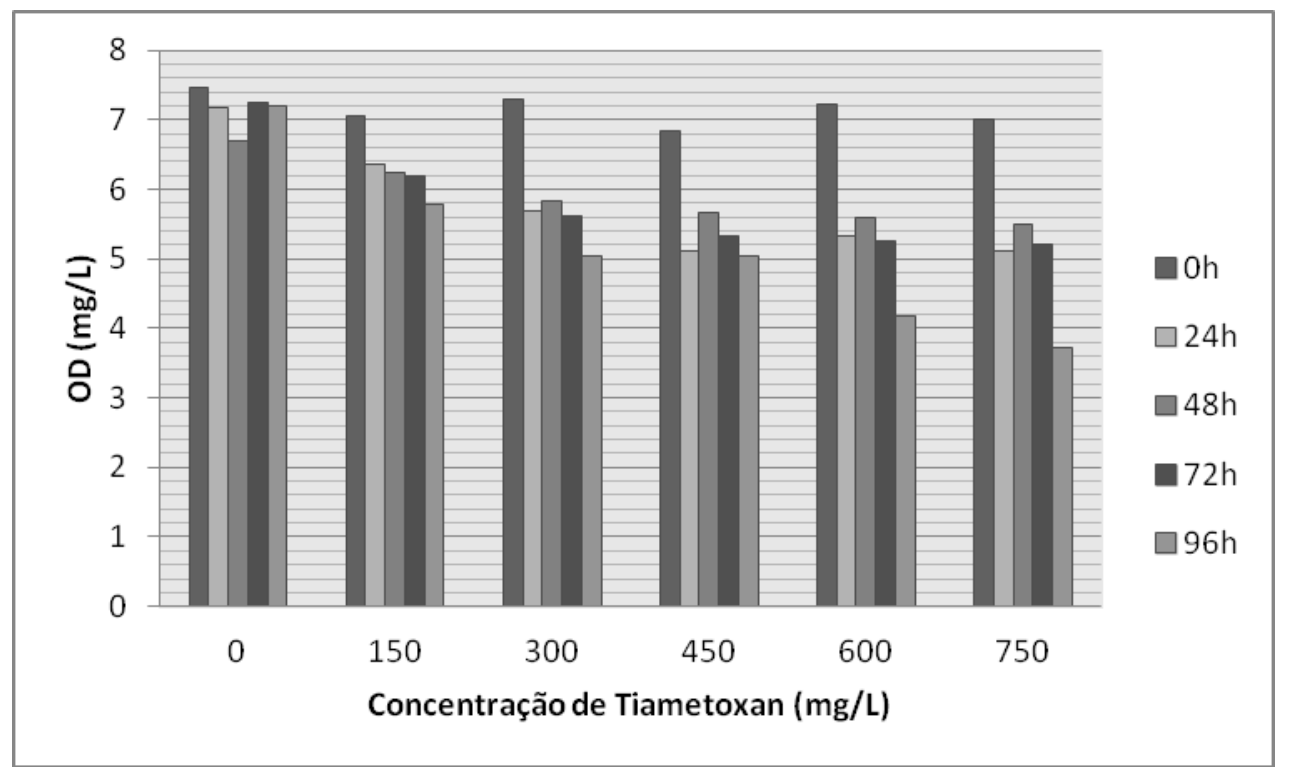

Figura 2. Variação do oxigênio dissolvido (OD) na água em função do tempo de exposição ao inseticida, para diferentes concentrações de tiametoxam.

Nas Fig. 1 e 2, observa-se que, embora tenha havido uma redução dos valores de $\mathrm{pH}$ e OD da água, em função do tempo de exposição ao inseticida, nas diferentes concentrações de tiametoxam, não houve uma correlação linear entre essas variáveis.

O valor mais baixo de OD foi de $3,7 \mathrm{mg} / \mathrm{L}$ após 96 horas de exposição a $750 \mathrm{mg} / \mathrm{L}$ do inseticida; este foi o único valor abaixo do recomendado, porém acredita-se que essa baixa concentração de OD não tenha sido a causa da mortalidade ocorrida nesse tanque, tendo em vista a rusticidade da espécie em estudo (Kubitza, 2000).

Carraschi (2014) também encontrou uma baixa concentração de OD em testes de toxicidade aguda nos peixes Mato Grosso e Pacu e no caramujo Pomacea, sendo observados valores de 
OD que variaram de $5,6 \mathrm{mg} / \mathrm{L}$ no controle a $0,60 \mathrm{mg} / \mathrm{L}$ após $48 \mathrm{~h}$ de exposição a $60 \mathrm{mg} / \mathrm{L}$ de tiametoxam em peixes da espécie Mato Grosso, e em Pacus o OD variou de $3,11 \mathrm{mg} / \mathrm{L}$ no controle a $0,40 \mathrm{mg} / \mathrm{L}$ após 24 horas de exposição a $35 \mathrm{mg} / \mathrm{L}$ do inseticida. A autora atribui essa diminuição à ação do inseticida e também ao consumo do oxigênio pelos organismos testes.

Em relação à mortalidade, no entanto, ao final das 96 horas de exposição, pode-se observar uma ocorrência de 2,5\% no grupo controle, $10 \%$ no exposto a $150 \mathrm{mg} / \mathrm{L}, 32 \%$ em $300 \mathrm{mg} / \mathrm{L}, 87,5 \%$ em $450 \mathrm{mg} / \mathrm{L}$, $95 \%$ em $600 \mathrm{mg} / \mathrm{L}$ e $100 \%$ em 750mg/L (Fig.3), indicando um crescimento linear da mortalidade à medida que se aumentou a concentração do inseticida ( $\mathrm{Y}=22,8 \mathrm{x}$ - 25,5, com $\left.\mathrm{R}^{2}=0,91\right)$. Carraschi (2014) também encontrou um aumento linear da mortalidade tanto para o Mato Grosso quanto para o Pacu.

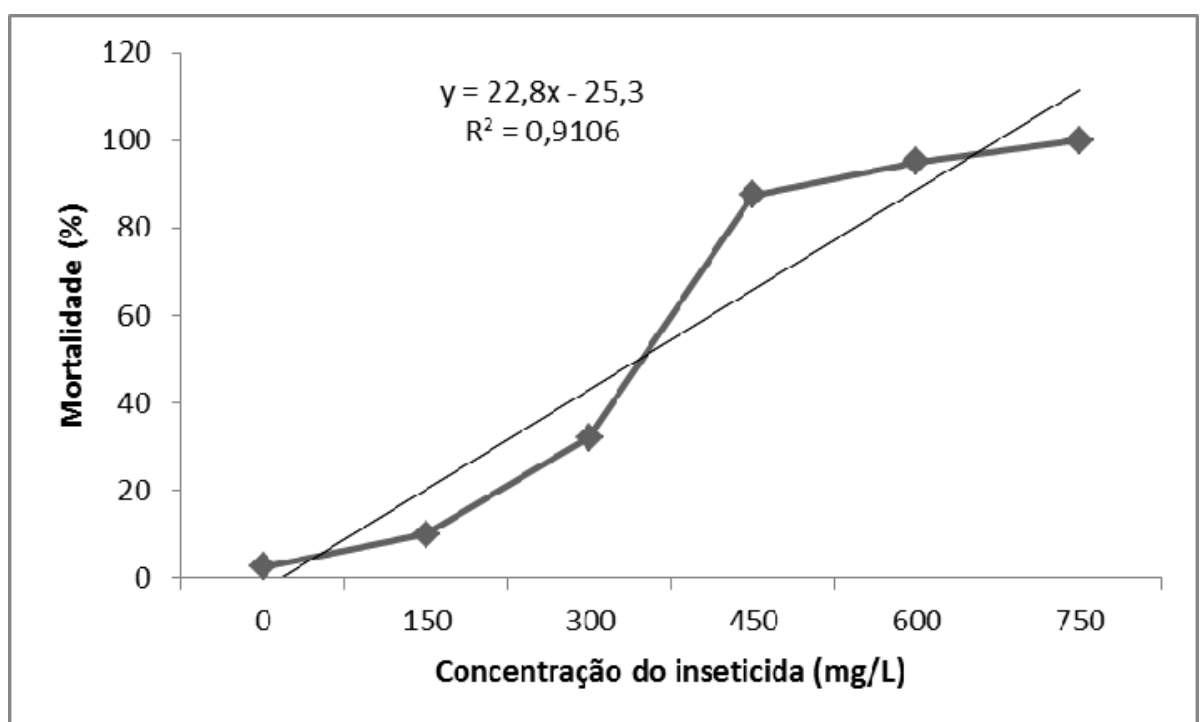

Figura 3. Mortalidade de alevinos de tilápia-do-nilo expostos a diferentes concentrações de tiametoxam por 96 horas.

Assim, apesar de o inseticida ter afetado a qualidade da água, parece não ser esta a razão da mortalidade dos peixes, que pode ser mais atribuída à ação deletéria do inseticida.

Os valores de CL50\%, calculados em ppm do produto comercial total, em 24, 48, 72 e 96 horas, e seus limites de confiança foram, respectivamente: $430,25 \quad(378,65 \quad-\quad 488,88)$; $415,39 \quad(361,88$ - 476,81); 410,60 (358,04 $470,87)$ e $322,08(288,79-359,21)$. A CL50\% 96h do Actara ${ }^{\circledR}$ para alevinos de tilápia foi de 322,08 ppm, sendo considerado praticamente não tóxico $(>100 \mathrm{mg} / \mathrm{L})$ de acordo com Zucker (1985).

A CL50\% apresentada foi calculada baseada no produto comercial que tem apenas $25 \%$ do ingrediente ativo, portanto, se extrapolados os valores de CL50\% achados somente para a porcentagem referente ao ingrediente ativo, obter-se-á uma CL50\% de 80,52mg/L, que sai da faixa de praticamente não tóxico para pouco tóxico $(10<\mathrm{CL} 50<100 \mathrm{mg} / \mathrm{L})$ (Zucker, 1985). Se comparados com os valores encontrados por Carraschi et al. (2015) para Pacu e Mato Grosso, respectivamente, de $16,97 \mathrm{mg} / \mathrm{L}$ e $49,78 \mathrm{mg} / \mathrm{L}$, observa-se que a toxicidade para as três espécies encontram-se na mesma faixa de pouco tóxico. Barbee e Stout (2009) encontraram CL50\% maior que $100 \mathrm{mg} / \mathrm{L}$ para truta-arco-íris e maior que $114 \mathrm{mg} / \mathrm{L}$ para bluegill sunfish. De acordo com Anderson et al. (2015), poucas espécies de peixes têm sido estudadas quanto à sensibilidade a neonicotinóides. Os poucos trabalhos de toxicidade aguda com o cálculo de CL50\% encontrados demonstram a baixa toxicidade do produto. No entanto, alguns trabalhos relatam alterações não letais, mas que comprometem a homeostase dos animais (Bose et al., 2011; Roy e Nath, 2011; Georgieva et al., 2014). Apesar da baixa toxicidade do tiametoxam, no que diz 
respeito à CL50\%, esse inseticida deve ser mais bem avaliado quanto a efeitos subletais e crônicos, uma vez que esse produto comercial tem sido prospectado para o uso como agente terapêutico para peixes.

Outro aspecto avaliado é o risco ambiental do inseticida e, segundo Spadotto (2009), a avaliação de risco ambiental deve ser feita para o defensivo como produto formulado, considerando-se os dados do produto técnico com suas impurezas e dos produtos de degradação relevantes.

Assim, em relação aos riscos ecotoxicológicos, estudados por meio da avaliação de concentração ambiental estimada (CAE) e do quociente de risco $(\mathrm{QR})$, pode-se observar, com base nos dados da Tab. 1, que houve uma variação entre os resultados de $\mathrm{QR}$, a depender do método utilizado para o cálculo. No cenário de $2 \mathrm{~m}$ de profundidade, quando se utiliza a CL50\%, baseada no produto comercial, tem-se um QR de 3,10 considerado alto. Para uma profundidade de $0,3 \mathrm{~m}$, no entanto, o risco é baixo para o produto comercial com um QR de 0,002.

No entanto, se os cálculos forem feitos considerando-se o valor estimado do princípio ativo do inseticida, o QR para o tiametoxam será baixo, independentemente da profundidade utilizada nos cálculos. Quando considerada a CAE de $75 \mathrm{mg} / \mathrm{L}$, baseada na dose eficaz para controle de parasitas em Pacus, relatada por Carraschi et al. (2014), o risco é considerado médio para o produto comercial e alto para o ingrediente ativo, o que está de acordo com o encontrado pela mesma autora, que relatou alto risco ambiental do tiametoxam em todas as espécies estudadas: a planta aquática Lemna minor, o caramujo Pomaceacanaliculata, o microcrustáceo Daphnia magna e os peixes Hyphessobrycon eques e Piaractus mesopotamicus (Carraschi et al., 2015).

Tabela 1. Concentração ambiental estimada e quociente de risco para tilápia-do-nilo

\begin{tabular}{cccccc}
\hline Dose & Profundidade $(\mathrm{m})$ & $\mathrm{Cl} 50$ & $\mathrm{CAE}$ & QR & Risco \\
\hline $2000 \mathrm{~g} \mathrm{ia} / \mathrm{ha}$ & 0,3 & $322 \mathrm{mg} / \mathrm{L}($ Actara $)$ & 0,66 & 0,002 & Baixo \\
$2000 \mathrm{~g} \mathrm{ia} / \mathrm{ha}$ & 0,3 & $80,5 \mathrm{mg} / \mathrm{L}(\% \mathrm{ia})$ & 0,66 & 0,0081 & Baixo \\
$2000 \mathrm{~g} \mathrm{ia} / \mathrm{ha}$ & 2,0 & $322 \mathrm{mg} / \mathrm{L}($ Actara $)$ & 0,1 & 3,10 & Alto \\
$2000 \mathrm{~g} \mathrm{ia} / \mathrm{ha}$ & 2,0 & $80,5 \mathrm{mg} / \mathrm{L}(\% \mathrm{ia})$ & 0,1 & 0,0012 & Baixo \\
$75 \mathrm{mg} / \mathrm{L}$ & - & $322 \mathrm{mg} / \mathrm{L}($ Actara $)$ & 75 & 0,23 & Médio \\
$75 \mathrm{mg} / \mathrm{L}$ & - & $80,5 \mathrm{mg} / \mathrm{L}(\% \mathrm{ia})$ & 75 & 0,93 & Alto \\
\hline
\end{tabular}

Segundo Rebelo e Caldas (2014), quando o QR alcança valores altos, pode existir uma situação de risco potencial para organismos não alvos, podendo requerer uma ação regulatória. Os mesmos autores relatam ser o QR um método eficiente e barato de identificar situações de risco, permitindo decisões de gestão.

\section{CONCLUSÕES}

A CL50\% 96 horas do Actara 250WG ${ }^{\circledR}$, para alevinos de tilápias-do-nilo, foi de 322,08ppm, considerada praticamente não tóxica. Nesse período, a presença do produto na água ocasiona a diminuição do $\mathrm{pH}$ e do oxigênio dissolvido. $\mathrm{O}$ quociente de risco $(\mathrm{QR})$ varia de baixo a alto de acordo com a metodologia usada.

\section{AGRADECIMENTOS}

À Fundação de Amparo à Ciência e Tecnologia do Estado de Pernambuco (Facepe), pela bolsa concedida à primeira autora (IBPG -0903$5.05 / 11)$.

\section{REFERÊNCIAS}

ANDERSON, J.C.; DUBETZ, C.; PALACE, V.P. Neonicotinoids in the Canadian aquatic environment: a literature review on current use products with a focus on fate, exposure, and biological effects. Sci. Total Environ., v.505, p.409-422, 2015.

BARBEE, G.C.; STOUT, M.J. Comparative acute toxicity of neonicotinoid and pyretroid insecticides to non-target crayfish (Procambarusclarkii) associated with ricecrayfish crop rotations. Pest. Manag. Sci., v.65, p.1250-1256, 2009. 
BOSE, S.; NATH, S; SAHANA, S.S. Toxic impact of tiametoxam on the growth performance and liver protein concentration of a freshwater fish oreochromis niloticus (trewavas). Indian J. Fundam. Appl. Life Sci., v.1, p.274280, 2011.

BRASIL. Ministério do Meio Ambiente. Conselho Nacional do Meio Ambiente. Resolução n.357. de 17 de março de 2005. Dispõe sobre a classificação dos corpos de água e diretrizes ambientais para o seu enquadramento, bem como estabelece as condições e padrões de lançamento de efluentes, e dá outras providências. Diário Oficial da União, Brasília, 18 mar. 2005. p.58-63.

CARRASCHI, S.P. Ecotoxicidade: segurança clínica e eficácia de fármacos em jovens de pacu (Piaractus mesopotamicus). 2014. 101f. Tese (Doutorado em Aquicultura) - Centro de Aquicultura, Universidade Estadual Paulista, Jaboticabal, SP.

CARRASCHI, S.P.; BARBUIO, R.; IKEFUTI, C.V. et al. Effectiveness of therapeutic agents in disease treatment in Piaractus mesopotamicus. Aquaculture, v.431, p.124-128, 2014.

CARRASCHI, S.P; FLORÊNCIO, T.; GARLICH, N. et al. Ecotoxicology of drugs used in fish disease treatment. J. Environ. Chem. Ecotoxicol., v.7, p.31-36, 2015.

ECOTOXICOLOGIA aquática: toxicidade aguda - método de ensaio com peixes. Rio de Janeiro: ABNT, 2011. (NBR 15088).

GEORGIEVA, E.; STOYANOVA, S.; VELCHEVA, I. et al. Histopathological alterations in common carp (Cyprinus carpio L.) gills caused by Thiamethoxam. Braz. Arch. Biol. Technol., v.57, p.991-996, 2014.

GRAZZIERO, D.L.P. Misturas de agrotóxicos em tanque nas propriedades agrícolas do Brasil. Planta Daninha, v.33, p.83-92, 2015.

GRISOLIA, C.K. Agrotóxicos: mutações, câncer e reprodução. Brasília: Universidade de Brasília, 2005. 392p.

HAMILTON, M.A.; RUSSO, R.C.; THURSTON, V. TrimedSperman-karber method for estimating medial lethal concentrations in toxicology bioassays. Environ. Sci. Technol., v.7, p.714-719, 1977.
KUBITZA, F. Tilápia: tecnologia e planejamento na produção comercial. Jundiaí: Acqua \& Imagem, 2000. 285p.

KUBITZA, F.; KUBITZA, L.M.M. Qualidade da água, sistemas de cultivo, planejamento da produção, manejo nutricional e alimentar e sanidade. Panorama Aquicul., v.10, p.44-53, 2000.

LOMBARDI, J.V. Fundamentos de toxicologia aquática. In: RANZANI-PAIVA, M.J.T.; TAKEMOTO, R.M.; LIZAMA, M.A.P. Sanidade de organismos aquáticos. São Paulo: Varela, 2004. p.263-272.

LONDRES, F. Agrotóxicos no Brasil: um guia para ação em defesa da vida. Rio de Janeiro: ASPTA, 2011. 190p.

MAIENFISCH, P.; ANGST, M.; BRANDL, F. et al. Chemistry and biology of thiamethoxam: a second generation neonicotinoid. Pest. Manag. Sci., v.57, p.906-913, 2001.

MANRIQUE, W.G.; FIQUEIREGO, M.A.P.; MACHADO-NETO, J.G. Dissipation and environmental risk of fipronil on aquatic environment. Biologist (Lima), v.11, p.107-117, 2013.

PROPOSED guidelines for ecological risk assessment. Washington: USEPA, 1996. 188p.

REBELO, R.M.; CALDAS, E.D. Avaliação de risco ambiental de ambientes aquáticos afetados pelo uso de agrotóxicos. Quim. Nova, v.37, p.1199-1208, 2014.

RIGOTTO, R.M.; VASCONCELOS, D.P.; ROCHA, M.M. Uso de agrotóxicos no Brasil e problemas para a saúde pública. Cad. Saúde Pública., v.30, p.1-3, 2014.

ROY, B.; NATH, S. Some haematological investigations on Oreochromis niloticus (Trewavas) following exposure Thiamethoxam. Acta Zool. Lituanica, v.21, p.301-305, 2011.

SAS. Statistical analysis system. Versão 8.2.6. Cary: SAS, 2002.

SCORZA JÚNIOR, R.; RIGATANO, R.L.O. Sorção, degradação e lixiviação do inseticida tiametoxam em dois solos de Mato Grosso do Sul. Rev. Bras. Eng. Agríc. Ambiental, v.16, p.564-572, 2012. 
SILVA, G.S.; ALBUQUERQUE JR, E.C.; AMORIM JR, A.C. et al. Avaliação da potencial contaminação das águas superficiais e subterrâneas por agrotóxicos em áreas de produção de uva para exportação no vale do São Francisco. In: SIMPÓSIO ÍTALOBRASILEIRO DE ENGENHARIA SANITÁRIA E AMBIENTAL, 12., 2014 Natal. Anais... Natal: ABES, 2014. p.1-7.

SISTEMA de agrotóxicos fitossanitários. 2012. Disponível em: $<\underline{\mathrm{http}: / /}$ agrofit.agricultura.gov.br $>$. Acessado em: 18 jul. 2012.

SPADOTTO, C.A. Avaliação de riscos ambientais do uso de defensivos agrícolas para a qualidade da água. Hortic. Bras., v.27, p.40604070, 2009.
TAVARES, D.A. Análise morfológica e imunocitoquímica do cérebro de abelhas africanizadas ApisMellifera após exposição à doses subletais do inseticida Tiametoxam. 2011. 75f. Dissertação (Mestrado em Biologia Celular e Molecular) - Instituto de Biociências de Rio Claro, Universidade Estadual Paulista, Rio Claro, SP.

U־GURLU, P.; ÜNLÜ, E.; SATAR, E.I. The toxicological effects of thiamethoxam on Gammarus kischineffensis (Schellenberg 1937) (Crustacea: Amphipoda). Environ. Toxicol. Pharmacol., v.39, p.720-726, 2015.

ZUCKER, E. Standard evaluation procedure: acute toxicity test for freshwater fish. Washington: USEPA, 1985. 17p. 